(1)

CrossMark

\title{
Bilateral hypoglossal nerve stimulation for treatment of adult obstructive sleep apnoea
}

\author{
Peter R. Eastwood (10), ${ }^{1,2}$, Maree Barnes ${ }^{3,4}$, Stuart G. MacKay 5,6,7,8, \\ John R. Wheatley ${ }^{9,10,11}$, David R. Hillman ${ }^{1,2}$, Xuân-Lan Nguyên ${ }^{12,13}$, \\ Richard Lewis ${ }^{14,15}$, Matthew C. Campbell ${ }^{3,4}$, Boris Pételle ${ }^{16}$, \\ Jennifer H. Walsh ${ }^{1,2}$, Andrew C. Jones ${ }^{5,6,7}$, Carsten E. Palme ${ }^{10,17}$, Alain Bizon ${ }^{18}$, \\ Nicole Meslier ${ }^{19,20}$, Chloé Bertolus ${ }^{21,22}$, Kathleen J. Maddison ${ }^{1,2}$, \\ Laurent Laccourreye $^{18}$, Guillaume Raux ${ }^{23}$, Katleen Denoncin ${ }^{23}$, \\ Valérie Attali ${ }^{13,21}$, Frédéric Gagnadoux ${ }^{19,20}$ and Sandrine H. Launois ${ }^{12,13}$
}

@ERSpublications

A new method of hypoglossal nerve stimulation to treat sleep apnoea does so bilaterally via an implanted neurostimulator activated externally. Its simplicity and relative non-invasiveness have not compromised its effectiveness relative to older methods. http://bit.ly/2lDCeif

Cite this article as: Eastwood PR, Barnes M, MacKay SG, et al. Bilateral hypoglossal nerve stimulation for treatment of adult obstructive sleep apnoea. Eur Respir J 2020; 55: 1901320 [https://doi.org/10.1183/ 13993003.01320-2019].

\section{ABSTRACT}

Background and aim: Hypoglossal nerve stimulation (HNS) decreases obstructive sleep apnoea (OSA) severity via genioglossus muscle activation and decreased upper airway collapsibility. This study assessed the safety and effectiveness at 6 months post-implantation of a novel device delivering bilateral HNS via a small implanted electrode activated by a unit worn externally, to treat OSA: the Genio ${ }^{\mathrm{TM}}$ system.

Methods: This prospective, open-label, non-randomised, single-arm treatment study was conducted at eight centres in three countries (Australia, France and the UK). Primary outcomes were incidence of device-related serious adverse events and change in the apnoea-hypopnoea index (AHI). The secondary outcome was the change in the $4 \%$ oxygen desaturation index (ODI). Additional outcomes included measures of sleepiness, quality of life, snoring and device use. This trial was registered with ClinicalTrials.gov, number NCT03048604.

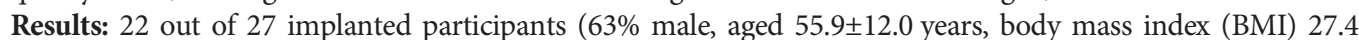
$\pm 3.0 \mathrm{~kg} \cdot \mathrm{m}^{-2}$ ) completed the protocol. At 6 months BMI was unchanged ( $\mathrm{p}=0.85$ ); AHI decreased from 23.7 \pm 12.2 to $12.9 \pm 10.1$ events $\cdot h^{-1}$, a mean change of 10.8 events $^{-1} h^{-1}(p<0.001)$; and ODI decreased from 19.1 \pm 11.2 to $9.8 \pm 6.9$ events $\cdot h^{-1}$, a mean change of 9.3 events. $h^{-1}(\mathrm{p}<0.001)$. Daytime sleepiness (Epworth Sleepiness Scale; $\mathrm{p}=0.01$ ) and sleep-related quality of life (Functional Outcomes of Sleep Questionnaire-10; $\mathrm{p}=0.02$ ) both improved significantly. The number of bed partners reporting loud, very intense snoring, or leaving the bedroom due to participant snoring decreased from $96 \%$ to $35 \%$. $91 \%$ of participants reported device use $>5$ days per week, and $77 \%$ reported use for $>5$ h per night. No device-related serious adverse events occurred during the 6-month post-implantation period.

Conclusions: Bilateral HNS using the Genio ${ }^{\mathrm{TM}}$ system reduces OSA severity and improves quality of life without device-related complications. The results are comparable with previously published HNS systems despite minimal implanted components and a simple stimulation algorithm.

This article has supplementary material available from erj.ersjournals.com

This study is registered with ClinicalTrials.gov identifier: NCT03048604.

Received: 4 July 2019 | Accepted after revision: 21 Sept 2019

Copyright $\odot$ ERS 2020 This article is open access and distributed under the terms of the Creative Commons Attribution Non-Commercial Licence 4.0. 


\section{Introduction}

Obstructive sleep apnoea (OSA) is a disorder characterised by recurrent episodes of decreased (hypopnoea) or absent (apnoea) inspiratory airflow during sleep. The primary mechanism underlying these events is a sleep-related decrease in pharyngeal muscle activity, which causes narrowing and collapse of the airway in predisposed individuals. The resultant intermittent episodes of arterial oxygen desaturation and repeated disruption of sleep cause excessive daytime sleepiness and other medical comorbidities such as hypertension, depression and stroke $[1,2]$.

The goal of OSA treatment is to prevent airway narrowing and/or collapse in order to maintain optimal breathing during sleep, to reduce comorbidities and to relieve associated symptoms. Positive airway pressure (PAP) is recognised as the primary treatment for patients with moderate-to-severe OSA. PAP involves the delivery of air under pressure to the pharynx via a well-fitting mask. This pressure acts as a pneumatic splint, holding the airway open and preventing its collapse. Although a highly efficacious treatment, patients are often uncomfortable using the device and adherence to therapy remains problematic [3, 4]. For this reason, there is substantial interest in developing alternative treatments for OSA. These include hypoglossal nerve stimulation (HNS), which modulates upper airway collapsibility though neural stimulation of the genioglossus muscle [5].

Since the first successful use of a HNS system to treat OSA was reported in 2001 [6], three HNS systems have been CE-marked. Apnex Medical published the first feasibility study in 2011, reporting a significant decrease in OSA severity and symptoms following implantation with their system [7], but this device did not enter clinical practice because a pivotal study failed to show a between-group difference in the reduction of sleep apnoea, owing to unanticipated improvements in the control group (ClinicalTrials.gov number NCT01446601) [8]. Inspire Medical Systems currently manufactures the only United States Food and Drug Administration-approved HNS device for OSA. This device is an implantable, pacemaker-like pulse generator with a sensing lead placed between the external and internal intercostal muscles to detect breathing effort, and a stimulation lead connected to a cuff electrode wrapped around one (unilateral) hypoglossal nerve [9]. A 60-month outcome study, the STAR trial, reported a significant decrease in OSA severity and symptoms [10]. ImThera Medical has developed the aura6000 ${ }^{\mathrm{TM}}$ system, another HNS device. This system does not include a sensing lead and stimulates the hypoglossal nerve with six electrodes at a more proximal location, co-activating the tongue protrusors and retractors (using different stimulation vectors) to stiffen the posterior aspect of the tongue and pharyngeal walls to open the airway [11]. Improvements in OSA severity and symptoms have been reported at 6 months following implantation [12].

This article presents the results of a multicentre, prospective, open-label, non-randomised, single-arm OSA treatment study of a novel HNS device, the Genio ${ }^{\text {TM }}$ system. It differs favourably from previous HNS devices as it does not require any leads (connective wires between the sensor/cuff electrodes and the pulse generator) and only one incision is required without any tunnelling. Furthermore, stimulation is delivered bilaterally and controlled from an externally worn unit that activates a small implanted battery-free submental stimulator at a predetermined, adjustable rate and duty cycle. This study, the BLAST OSA study (Bilateral Hypoglossal Nerve Stimulation for Treatment of Obstructive Sleep Apnoea) was undertaken to

Affiliations: ${ }^{1}$ Centre for Sleep Science, School of Human Sciences, University of Western Australia, Perth, Australia. ${ }^{2}$ West Australian Sleep Disorders Research Institute, Sir Charles Gairdner Hospital, Perth, Australia. ${ }^{3}$ Institute for Breathing and Sleep, Austin Hospital, Heidelberg, Australia. ${ }^{4}$ University of Melbourne, Parkville, Australia. ${ }^{5}$ Illawarra ENT Head and Neck Clinic, Wollongong, Australia. 'Wollongong Hospital, Illawarra Shoalhaven Local Health District (ISLHD), Wollongong, Australia. ${ }^{7}$ Graduate School of Medicine, University of Wollongong, Wollongong, Australia. ${ }^{8}$ Woolcock Institute of Medical Research, Glebe, Australia. ${ }^{9}$ Dept of Respiratory and Sleep Medicine, Westmead Hospital, Westmead, Australia. ${ }^{10}$ University of Sydney at Westmead Hospital, Westmead, Australia. ${ }^{11}$ Ludwig Engel Centre for Respiratory Research, The Westmead Institute for Medical Research, Westmead, Australia. ${ }^{12}$ Unité de Somnologie et Fonction Respiratoire, Hopital St Antoine, Paris, France. ${ }^{13}$ Sorbonne Université, INSERM, UMRS1158 Neurophysiologie Respiratoire Expérimentale et Clinique, Paris, France. ${ }^{14}$ Dept Otolaryngology, Head and Neck Surgery, Royal Perth Hospital, Perth, Australia. ${ }^{15}$ Hollywood Private Hospital, Perth, Australia. ${ }^{16}$ Service ORL Chirurgie de la Face et du Cou, Hôpital Tenon, AP-HP, Paris, Sorbonne Université, Paris, France. ${ }^{17}$ The Dept of Otolaryngology Head Neck Surgery, Westmead Hospital, Westmead, Australia. ${ }^{18}$ Dept Otolaryngology, Head and Neck Surgery, University Hospital of Angers, Angers, France. ${ }^{19}$ Dept of Respiratory and Sleep Medicine, University Hospital of Angers, Angers, France. ${ }^{20}$ INSERM UMR 1063 "SOPAM", University of Angers, Angers, France. ${ }^{21}$ AP-HP, Groupe Hospitalier Pitié-Salpêtrière Charles Foix, Service des Pathologies du Sommeil (Département "R3S"), Paris, France. ${ }^{22} \mathrm{AP}-\mathrm{HP}$, Groupe Hospitalier Pitié-Salpêtrière Charles Foix, Service de Stomatologie et Chirurgie Maxillo-faciale, Paris, France. ${ }^{23}$ Nyxoah, S.A., Mont-Saint-Guibert, Belgium.

Correspondence: Peter R. Eastwood, Centre for Sleep Science, School of Human Sciences, University of Western Australia, Crawley, Western Australia 6009, Australia. E-mail: peter.eastwooddahealth.wa.gov.au 
evaluate the safety and effectiveness of the Genio ${ }^{\text {TM }}$ system over a period of 6 months in adult participants with moderate-to-severe OSA.

\section{Methods}

\section{Participants}

Participants were recruited from sleep clinics and otolaryngology practices, and were eligible for implantation if they met the following criteria: age $21-75$ years; body mass index (BMI) $\leqslant 32 \mathrm{~kg} \cdot \mathrm{m}^{-2}$; obstructive apnoea-hypopnoea index (AHI) 20-60 events. $\mathrm{h}^{-1}$ and combined central and mixed AHI $<10$ events $\cdot \mathrm{h}^{-1}$; no positional OSA (defined as nonsupine AHI $<10$ events $\mathrm{h}^{-1}$ and supine $\mathrm{AHI} \geqslant$ non-supine-AHI $\times 2$ ); absence of soft palate complete concentric collapse (CCC) during drug-induced sleep endoscopy [13]; and had not tolerated or accepted PAP treatments. For a complete list of inclusion and exclusion criteria see supplementary table S1. An additional inclusion criterion for French sites only was "participants who do not tolerate mandibular advancement devices".

\section{Study overview and design}

The study design was a multicentre, prospective, open-label, non-randomised, single-arm treatment study. Potential participants were provided with information about the study. If they agreed to take part, they underwent testing to confirm full eligibility during an 8-week period, during which baseline measurements (including baseline polysomnography (PSG)) were obtained. If eligibility was confirmed, participants were surgically implanted with the Genio ${ }^{\mathrm{TM}}$ system (Nyxoah SA, Mont-Saint-Guibert, Belgium) under general anaesthesia. The procedure required making a small incision above the hyoid bone and dissecting though the platysma, mylohyoid and geniohyoid muscles to the genioglossus muscle. The hypoglossal nerve branches were then identified and the stimulation unit (figure 1a) sutured in place (more detail given in Study device section). The device was activated 4-6 weeks after implantation, titrated (optimised) at follow-up visits at 2, 3 and 4 months, and outcome measurements obtained at a 6-month follow-up visit (figure 2) with fixed therapeutic settings on full-night PSG.

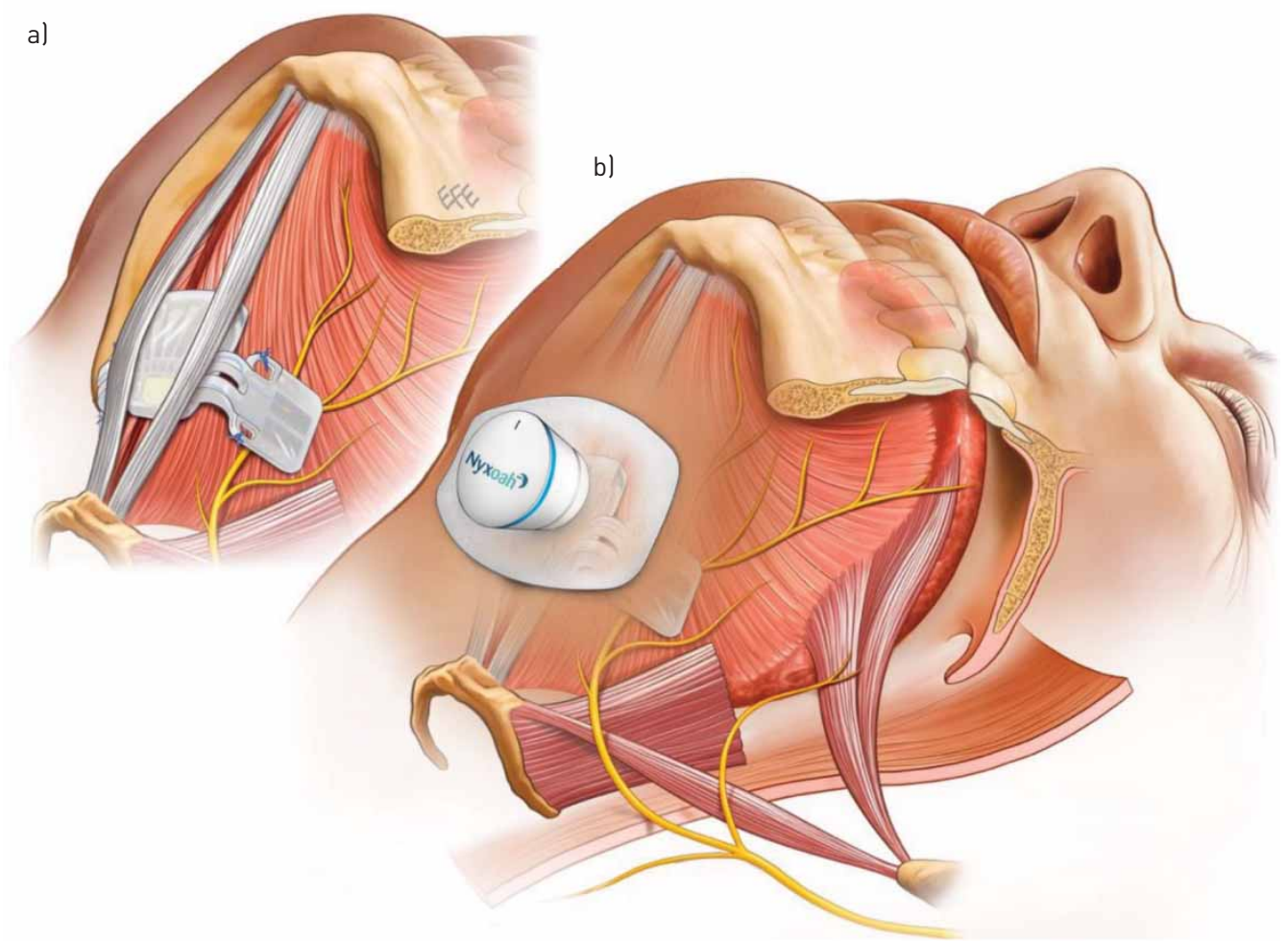

FIGURE 1 Submental musculature showing a) the implanted stimulator straddling the genioglossus muscles and hypoglossal nerve branches bilaterally and b) the disposable patch and activation unit. The images are for illustrational purposes only and it should be noted that the surgical anatomy might differ from person to person, thereby requiring adjustment to the specific placement of the implanted stimulator over the hypoglossal nerves. 
FIGURE 2 Flow diagram showing study enrolment and participant progress. PSG: polysomnography; DISE: drug-induced sleep endoscopy.

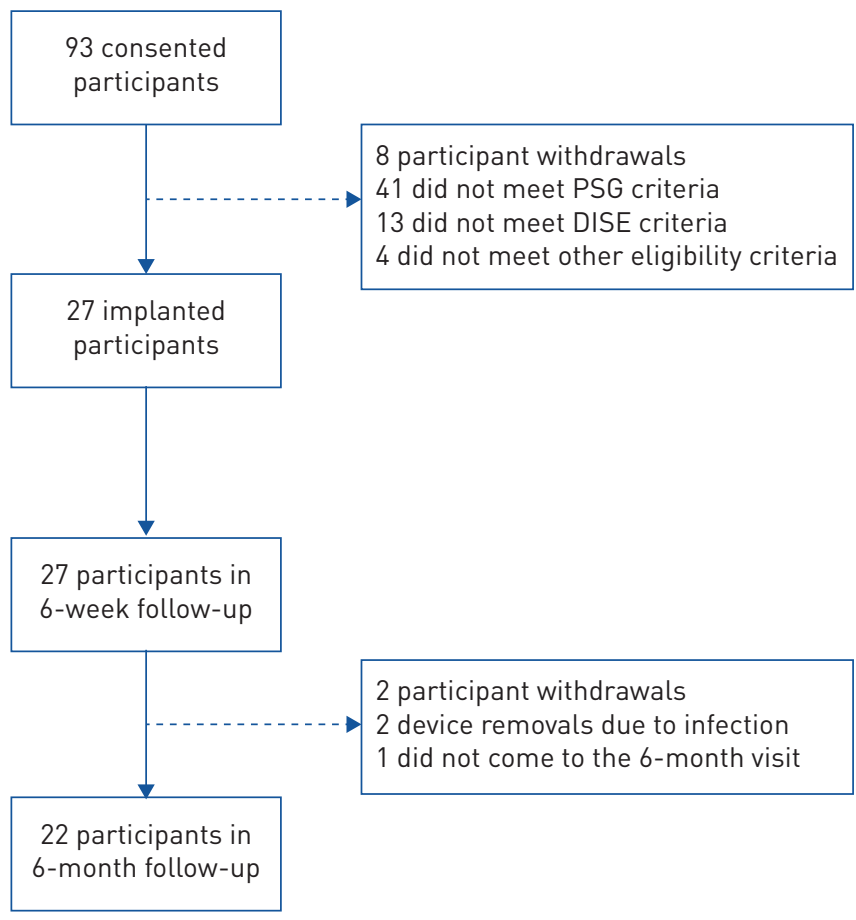

\section{Outcomes}

The primary outcome measures were the incidence of device-related serious adverse events (SAEs) and the change in AHI. The secondary outcome measure was the change in the $4 \%$ oxygen desaturation index (ODI). Additional outcome measures were changes in time spent at an oxygen desaturation <90\%; sleep efficiency; sleep fragmentation using the arousal index; daytime sleepiness using the Epworth Sleepiness Scale (ESS); sleep-related quality of life using the Functional Outcomes of Sleep Questionnaire (FOSQ)-10; partner-reported snoring; and the number of participants considered "responders" to the therapy, defined using the established standard for similar studies of surgical outcomes in OSA of $\geqslant 50 \%$ reduction in mean AHI and an AHI of $<20$ events. $h^{-1}$ [14]. An objective measurement of the time spent using the device each night could not be obtained with the current generation of the device. For this reason, nightly usage of the Genio ${ }^{\mathrm{TM}}$ system was evaluated though a usability questionnaire completed by the participants at 6 months of the number of hours used per night and the number of nights used per week.

\section{Study device}

The Genio ${ }^{\mathrm{TM}}$ system consists of a stimulation unit (figure 1a) surgically implanted in the submental region, positioned over the genioglossus muscles with its stimulating electrodes proximate to both the left and right hypoglossal nerve branches. This electrode positioning was adjusted with the aid of a nerve integrity monitor system. In order to stimulate the nerves, the implanted stimulation unit receives energy pulses transmitted transdermally from an external activation unit, which can be attached to an adhesive disposable patch and which is placed under the chin by the participant prior to going to sleep (figure 1b). These are removed by the participant in the morning, the disposable patch is discarded, and the activation unit recharged for its next use (supplementary figure $\mathrm{S} 1$ ).

The activation unit holds participant-specific stimulation parameters that are preprogrammed and which are adjusted wirelessly. Device programming and adjustments occurred during awake titrations as well as during in-lab PSG studies performed prior to the 6-month end-point visit. During PSG studies stimulation parameters were refined until settings were obtained that did not wake the participant and maintained upper airway patency and oxyhaemoglobin saturation. Stimulation "on" time (train length) and stimulation "off" time (train interval) was preprogrammed based on each participant's breathing frequency measured during unobstructed breathing when asleep (supplementary figure S2). The device is a constant voltage source with intensity of the stimulation mainly controlled by the pulse amplitude, represented by the percentage of the maximal amount of energy that can be delivered to the nerve by the implanted stimulator considering its relative position and the impedance between the electrodes and the nerve (supplementary table S2). Most participants needed time to reach tolerance of stimulation therapy levels; hence optimisation took up to 4 months. 


\section{Sleep recordings and scoring}

All PSG results in this publication were generated from sleep studies scored by an independent core laboratory (Registered Sleepers, Leicester, NC, USA). Participants were included in the trial based on 2014 American Academy of Sleep Medicine (AASM) recommended scoring guidelines [15]. However, to permit more direct comparison with available literature [9], all results presented in this paper are based on the 2014 AASM acceptable scoring guidelines (additional details can be found in the supplementary material) [15].

\section{Statistical analysis}

In order to detect a clinically meaningful reduction of $\geqslant 15$ events $\mathrm{h}^{-1}$ in $\mathrm{AHI}$ with $90 \%$ power at a $5 \%$ level of significance, and assuming a standard deviation of 20 events. $\mathrm{h}^{-1}$, a total sample size of 21 subjects was required to test the null hypothesis. Allowing for a 15\% drop-out, 25 subjects were included.

The changes from baseline to 6 months after surgery in AHI, ODI, the ESS and FOSQ-10 were calculated for each participant. $p$-values from a paired t-test were provided for the different measures and all data were presented as mean $\pm S D$, unless otherwise stated. Safety-related analyses were performed on an intention-to-treat basis and included all participants who underwent study procedures with data available for analysis $(\mathrm{n}=27)$. Modified intention-to-treat analyses were performed on all other measures by excluding two participants in whom no titration was performed (i.e. no PSG data available post-implant) and three participants who withdrew prior to the 6-month PSG study (i.e. $n=22$ ). Additional analyses were undertaken on a per-protocol basis in participants without any major protocol deviation and good compliance with the therapy $(\mathrm{n}=19)$ (additional details can be found in the supplementary material).

\section{Study oversight and approvals}

A clinical events committee independently reviewed any adverse events. The clinical events committee consisted of three experienced and recognised ear, nose and throat surgeons and sleep medicine specialists. All individuals provided written, informed consent prior to participation in the study, which was conducted in compliance with ISO14155:2011 and approved by the ethics committees at all centres. Additional details can be found in the supplementary material.

\section{Results}

\section{Participant characteristics}

Between April 2017 and February 2018, seven centres in France and Australia screened 93 participants into the study (one centre was activated in the UK, but did not enrol any participants) (supplementary table S3). 66 participants failed the screening after consent and did not receive an implant (supplementary table S4). The most common reasons for this were CCC at the soft palate and participant AHI results outside the allowed screening range (based on full-night PSG). 27 participants were implanted with the Genio $^{\mathrm{TM}}$ system (figure 2).

Among the 27 implanted participants, 22 reached the 6-month follow-up visit (figure 2). Two participants exited the study prior to the first post-implant PSG due to procedure-related infections. One participant was withdrawn from the study due to non-study-related behavioural issues. Another one should not have been implanted, since only limited HNS response was observed during the surgery, and was subsequently withdrawn from the study. Finally, one participant was withdrawn as they failed to return for the 6-month end-point visit despite numerous attempts from the centre to re-establish contact with the participant.

The demographics of the 27 participants implanted with the Genio ${ }^{\mathrm{TM}}$ system are presented in table 1 . The mean age was $55.9 \pm 12.0$ years; mean BMI $27.4 \pm 3.0 \mathrm{~kg} \cdot \mathrm{m}^{-2} ; 63.0 \%$ (17 out of 27 ) were male; and $88.9 \%(24$ out of 27) were Caucasian. In the 22 who reached the 6-month follow-up visit, BMI was unchanged when compared to baseline, being 27.73 and $27.67 \mathrm{~kg} \cdot \mathrm{m}^{-2}$, respectively $(\mathrm{p}=0.85)$.

\section{Primary outcomes}

No device-related SAEs occurred during the 6 months post-implantation. Three out of the 27 implanted participants experienced four SAEs related to the surgical procedure: three were local infections at the surgical site necessitating explantation of the devices at 2 and 3 months after implantation, including two participants at the same centre. The corresponding SAEs were resolved without further sequelae. The fourth procedure-related SAE was impaired swallowing, which led to a 1-day prolongation of implantation-related hospitalisation. This SAE resolved spontaneously without further sequelae. The most frequent procedure-related non-serious adverse events that occurred in implanted participants were impairment or painful swallowing (30\% of participants), dysarthia ( $26 \%$ of participants), haematoma (19\% of participants) and swelling or bruising around the incision site (19\% of participants). Among the device-related non-serious adverse events, $30 \%$ of participants experienced local skin irritation due to the disposable patch, which resolved in all cases except one, which remained present at the 6-month visit. The 
TABLE 1 Demographic and baseline characteristics

$\begin{array}{lcc}\text { Age years } & 55.9 \pm 12.0 & 58.5(32.5-74.7) \\ \text { Male } & 63(17 / 27) & \\ \text { BMI } \mathbf{~ g g} \cdot \mathbf{m}^{-2} & 27.4 \pm 3.0 & 28.1(20.7-32.3) \\ \text { BP systolic } \mathbf{m m H g} & 130.4 \pm 17.5 & 130.0(86.0-177.0) \\ \text { BP diastolic } \mathbf{m m H g} & 78.1 \pm 6.6 & 78.0(61.0-90.0) \\ \text { Neck circumference cm } & 39.0 \pm 4.2(\mathrm{n}=24) & 39.5(32.0-48.0) \\ \text { Caucasian } & 88.9(24 / 27) & \\ \text { Hispanic } & 11.1(3 / 27) & \end{array}$

Data are presented as mean $\pm \mathrm{SD}, \%(\mathrm{n} / \mathrm{N})$ or median (range), unless otherwise stated. $\mathrm{BMI}$ : body mass index; BP: blood pressure.

events of skin irritation were resolved without any treatment or with topical medication and, in two cases, temporarily suspending use of the disposable patch. Other non-serious device-related adverse events included tongue abrasion ( $11 \%$ of participants), tongue fasciculations ( $11 \%$ of participants) and discomfort due to electrical stimulation (11\% of participants) (supplementary table S5). There were no instances of extrusion of the stimulation unit or the sutures used to hold it in place.

Mean AHI decreased from baseline to the 6-month PSG from $23.7 \pm 12.2$ to $12.9 \pm 10.1$ events $^{-1} \mathrm{~h}^{-1}$ $(\mathrm{p}<0.0001)$ (table 2 and figure 3); the mean individual percentage decrease was $47.3 \%$ (median $48.6 \%$ ). When examined using a per-protocol analysis $(n=19)$ the mean AHI decreased from $22.2 \pm 12.0$ to $11.0 \pm 9.5$ events $\cdot \mathrm{h}^{-1}$ from baseline to the 6-month PSG, respectively $(\mathrm{p}<0.0001)$ (supplementary table S6 and supplementary figure S3); the mean individual percentage decrease was $51.4 \%$ (median $55.1 \%$ ). The responder rate was $50.0 \%$ (11 out of 22 ) for the modified intention-to-treat analysis and $57.9 \%$ (11 out of 19) for the per-protocol analysis. Additionally, the therapy resulted in 11 participants with a residual AHI $<15$ events. $\mathrm{h}^{-1}$, four participants with AHI $<10$ events. $\mathrm{h}^{-1}$ and three participants with AHI $<5$ events. $\mathrm{h}^{-1}$.

\section{Secondary outcome}

Mean ODI decreased from baseline to the 6-month PSG from 19.1 \pm 11.2 to $9.8 \pm 6.9$ events $\cdot \mathrm{h}^{-1}(\mathrm{p}<0.0001)$ (table 2 and figure 4); the mean individual percentage decrease was $43.3 \%$ (median $47.2 \%$ ). Using the per-protocol analysis $(n=19)$ the mean ODI decreased from $18.2 \pm 10.4$ to $8.0 \pm 5.4$ events $\cdot h^{-1}$, from baseline

TABLE 2 Outcome measures for modified intention-to-treat analyses

\begin{tabular}{|c|c|c|c|c|}
\hline & Baseline & 6 months & Mean difference $(95 \% \mathrm{CI})$ & p-value \\
\hline Subjects $\mathbf{n}$ & 22 & 22 & & \\
\hline \multicolumn{5}{|l|}{ Sleep disordered breathing } \\
\hline $\mathrm{AHI}$ events $\cdot \mathrm{h}^{-1}$ & $23.7 \pm 12.2$ & $12.9 \pm 10.1$ & $10.8(14.6-7.0)$ & $<0.0001$ \\
\hline ODI events $\cdot h^{-1}$ & $19.1 \pm 11.2$ & $9.8 \pm 6.9$ & $9.3(13.1-5.5)$ & $<0.0001$ \\
\hline $\mathrm{S}_{\mathrm{aO}_{2}}<90 \% \%$ time & $5.0 \pm 6.0$ & $2.1 \pm 3.0$ & $2.9(4.6-1.3)$ & 0.0015 \\
\hline Apnoea index events $\cdot \mathrm{h}^{-1}$ & $10.1 \pm 10.2$ & $5.6 \pm 8.4$ & $4.8(9.2-0.4)$ & 0.0334 \\
\hline Hypopnoea index events. $h^{-1}$ & $12.5 \pm 8.9$ & $7.6 \pm 6.2$ & $4.9(8.1-1.7)$ & 0.0049 \\
\hline \multicolumn{5}{|l|}{ Symptoms } \\
\hline ESS & $11.0 \pm 5.3^{\#}$ & $8.0 \pm 5.4$ & $3.0(5.7-0.8)$ & 0.0113 \\
\hline FOSQ-10 & $15.3 \pm 3.3$ & $17.2 \pm 3.0$ & $1.9(0.4-3.4)$ & 0.0157 \\
\hline \multicolumn{5}{|l|}{ Sleep architecture } \\
\hline Sleep efficiency \% & $84.0 \pm 10.8$ & $87.3 \pm 8.9$ & $3.2(0.01-6.4)$ & 0.0494 \\
\hline NREM stage $1 \%$ & $13.1 \pm 7.9$ & $8.2 \pm 4.0$ & $5.0(8.3-1.7)$ & 0.0053 \\
\hline NREM stage $2 \%$ & $60.9 \pm 8.7$ & $67.6 \pm 9.5$ & $6.7(2.2-11.3)$ & 0.0058 \\
\hline NREM stage $3 \%$ & $8.2 \pm 6.9$ & $3.5 \pm 4.3$ & $4.7(6.6-2.7)$ & $<0.001$ \\
\hline REM \% & $17.8 \pm 6.4$ & $20.7 \pm 7.3$ & $2.9(-0.3-6.2)$ & 0.0782 \\
\hline Arousal index events $\cdot \mathrm{h}^{-1}$ & $28.7 \pm 11.5$ & $16.0 \pm 8.0$ & $12.7(16.6-8.9)$ & $<0.0001$ \\
\hline \multicolumn{5}{|c|}{$\begin{array}{l}\text { Data are presented as mean } \pm \text { SD or mean }(95 \% \mathrm{Cl}) \text {, unless otherwise stated. AHI: apnoea-hypopnoea index; } \\
\text { ODI: } 4 \% \text { oxygen desaturation index; } \mathrm{S}_{\mathrm{aO}}<90 \% \text { : proportion of the night spent at an oxygen saturation }<90 \% \text {; } \\
\text { ESS: Epworth Sleepiness Scale; FOSQ-10: } 10 \text {-item Functional Outcomes of Sleep Questionnaire; NREM: } \\
\text { non-rapid eye movement; REM: rapid eye movement. }{ }^{\#}: \mathrm{n}=21 \text {. }\end{array}$} \\
\hline
\end{tabular}


FIGURE 3 Change in apnoeahypopnoea index (AHI) for each participant from baseline to 6 months post-implantation. Each line represents an individual participant using modified intention-to-treat analyses $(\mathrm{n}=22)$.

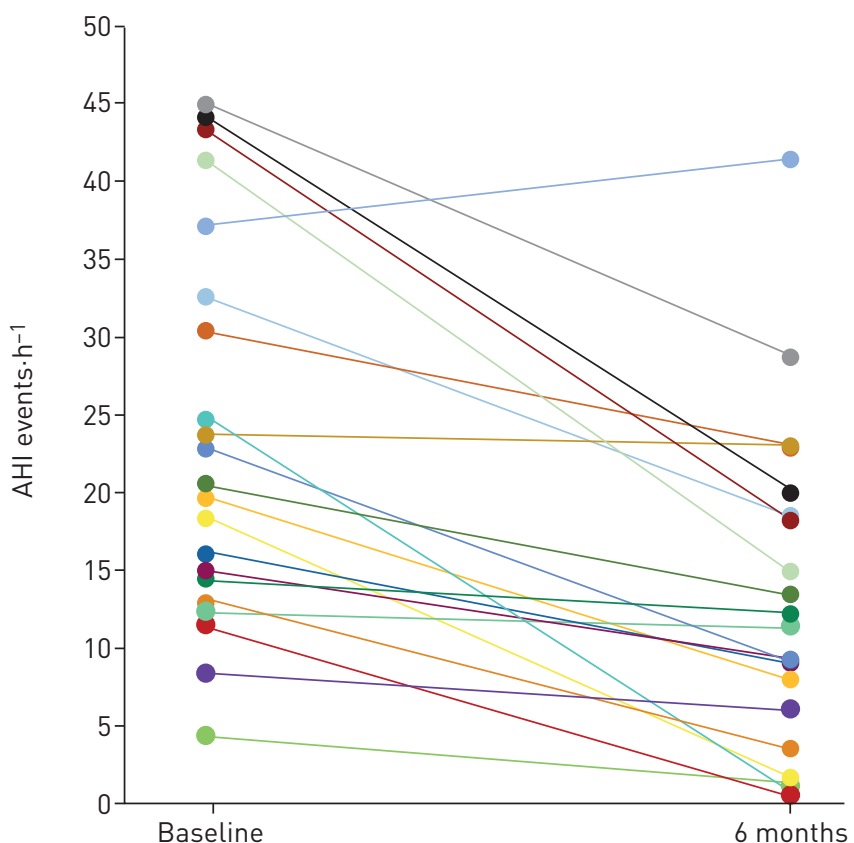

to the 6-month PSG, respectively $(\mathrm{p}<0.0001)$ (supplementary table S6 and supplementary figure S4); the mean individual percentage decrease was 50.6\% (median 56.0\%).

\section{Additional outcomes}

The ESS decreased from $11.0 \pm 5.3$ to $8.0 \pm 5.4$, a mean change of 3.3 (95\% CI $0.8-5.7$, median 1.0; $\mathrm{p}=0.0113)$, whereas the FOSQ-10 score increased from $15.3 \pm 3.3$ to $17.2 \pm 3.0$, a mean change of $1.9(95 \%$ CI 0.4-3.4, median 1.0; $\mathrm{p}=0.0157$ ) (table 2). The apnoea index, hypopnoea index, arousal index and time spent with proportion of the night spent at an oxygen saturation $<90 \%$ significantly decreased (all $p<0.05$ ) (table 2). Sleep efficiency increased, the proportion of the night spent in non-rapid eye movement (NREM) stage 1 and NREM stage 3 sleep decreased and the proportion of the night spent in NREM stage 2 and rapid-eye movement (REM) sleep increased (all p<0.05) (table 2). Bed partners reporting loud, very intense snoring, or leaving the bedroom due to partner snoring decreased from $96 \%$ at baseline to $35 \%$ at

FIGURE 4 Change in $4 \%$ oxygen desaturation index (ODI) for each participant from baseline to 6 months post-implantation. Each line represents an individual participant using modified intention-to-treat analyses $(\mathrm{n}=22)$.

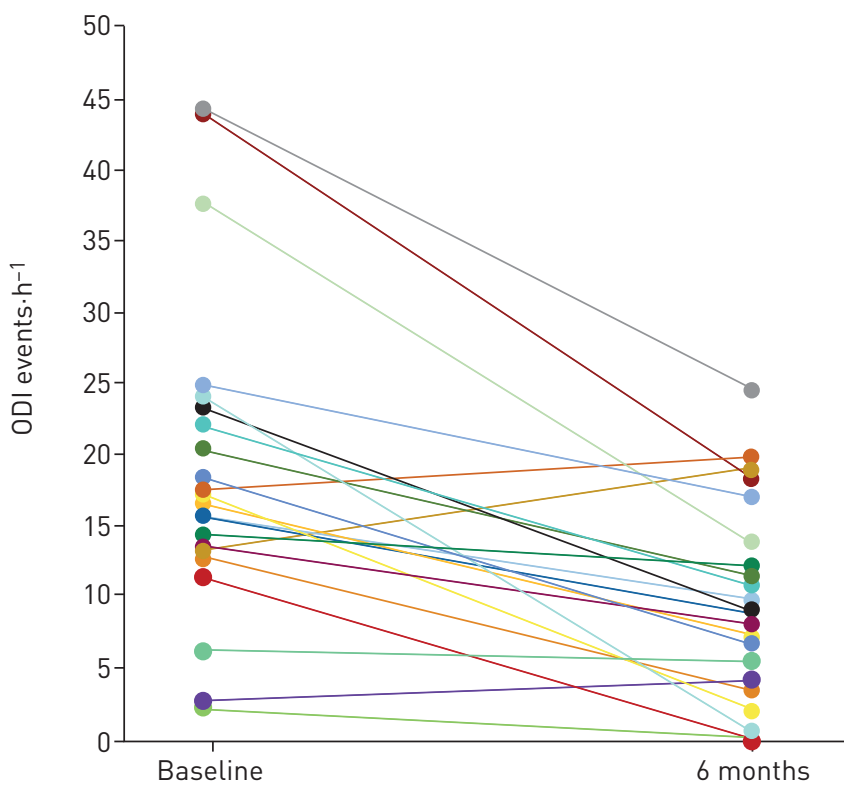


6 months post-implantation (supplementary table S7). Finally, at 6 months post-implantation $91 \%$ of participants reported using the Genio ${ }^{\mathrm{TM}}$ system $>5$ days a week and $77 \%$ reported a nightly use of $>5 \mathrm{~h}$ per night.

\section{Discussion}

The safety profile of the Genio ${ }^{\mathrm{TM}}$ system was favourable, given the absence of any device-related SAEs over the course of the study. The four procedure-related SAEs were resolved without further sequelae. This procedure-related SAE occurrence compares favourably with other HNS device reports [9, 12]. The two local infections requiring explantation of the device occurred at the one centre and were judged by the clinical events committee to be related to the surgical procedure rather than the device itself. The non-serious procedure-related AEs were anticipated with the type of upper airway surgery performed under general anaesthesia. All non-serious device-related adverse events, including local skin irritation due to the disposable patch, were resolved, except one where irritation remained evident at the 6-month visit. Stimulation could be initially experienced as uncomfortable to some participants, but often resolved with simple device parameter adjustments. Despite these minor side-effects, usage of the therapy was high, with $91 \%$ of participants using the system $>5$ days a week and $77 \%$ reporting using it $>5 \mathrm{~h}$ per night. This exceeds most reports of adherence to PAP treatment, with patient compliance ranging from $29 \%$ to $83 \%$ [3, 16-18], although recent data from patients undergoing standard clinical care but using current PAP technology suggests that short-term (90-day) adherence rates are $\sim 75 \%$ in individuals who accept PAP therapy [19].

There were improvements in the primary and secondary performance end-points at 6 months post-implantation. The mean individual percentage decrease in these measures was $47.3 \%$ and $43.3 \%$, respectively, for the modified intention-to-treat analyses $(n=22)$ and $51.4 \%$ and $50.6 \%$, respectively, for the per-protocol analyses $(n=19)$. The magnitude of change is similar to that reported by studies using other HNS devices, which range from $52 \%$ to $62 \%$ decrease in AHI and from $45 \%$ to $52 \%$ decrease in ODI $[7,9,21,22]$. The number of overnight titration visits to achieve these results is similar to that reported for other available HNS systems [23].

The improvements in objective measures of OSA severity were accompanied by improvements in symptoms. Specifically, the mean ESS score at the 6-month visit was below the threshold of 10 defined in the literature as being equivalent to a normal population [24]. In addition, although the 1.9-point increase in the FOSQ-10 score just failed to meet the two-point standard threshold for a clinically meaningful improvement quality of life [25] in the modified intention-to-treat analysis, the per-protocol analysis showed an increase of 2.5 points. This clinically significant improvement in quality of life is similar to improvements reported with other device usage $[7,9,22,26]$. These changes were accompanied by decreases in partner-reported snoring intensity and improvements in sleep architecture, specifically an increase in sleep efficiency and REM sleep, and a decrease in stage 1 sleep and in the number of arousals. Such changes are generally consistent with those reported in studies with other devices [7,27].

Compared to other implantable HNS devices the Genio ${ }^{\mathrm{TM}}$ system has four main differences. Firstly, rather than the unilateral stimulation offered by previous systems, the Genio ${ }^{\mathrm{TM}}$ system delivers bilateral HNS. This is achieved by the implantable stimulation unit that sits like a "saddle on a horse" over the genioglossus muscles near their insertion on the mandible, such that its stimulating electrodes face both the left and the right distal (medial) hypoglossal nerve branches. This approach, using paddle electrodes, differs from other systems which are based on unilateral stimulation of the hypoglossal nerve as the cuff electrode is positioned around only one hypoglossal nerve branch, usually the right $[7,9,12]$. While no studies have directly compared the effects on airway patency of bilateral versus unilateral HNS, several findings support the notion that bilateral stimulation might result in an improved response. For example, studies using unilateral HNS have reported that when tongue protrusion occurs in a more anterior motion (i.e. bilateral movement) rather than to the left or right, a better outcome is achieved [28, 29]. The improved response could be due to a number of factors including the difference in type of electrodes (paddle versus cuff), the applicable location of the cuff electrode on the hypoglossal nerve and resultant activation of different sets of pharyngeal muscles [30]; recruitment of genioglossus muscle fibres that receive innervation from the hypoglossal nerve on the contralateral side (i.e. cross-talk from right to left) [31]; or greater movement of the soft palate with forward motion of the tongue as a consequence of improved coupling of the palatoglossus muscle in the soft palate with the muscles of the lateral tongue body $[32,33]$. The more symmetrical muscle activation provided by the bilateral nature of the HNS delivered by the system has positive implications for patient comfort and functionality. Notably, as with other HNS devices, this device is designed to stimulate the hypoglossal nerve rather than the muscle directly. Indeed, the energy delivered by the device is insufficient to generate muscle activation though direct stimulation. 
A second difference between the Genio ${ }^{\mathrm{TM}}$ system and other HNS devices it that it stimulates only the genioglossus muscle, as a result of positioning the stimulating paddle electrodes close to the insertion of the distal hypoglossal nerve into the genioglossus muscle. In contrast, other systems use cuff electrodes placed around more proximal segments of the hypoglossal nerve resulting in stimulation of additional upper airway muscles with a consequent variety of movement patterns of the upper airway structures, including the tongue [28, 33-35]. Despite these differences in approach the magnitude of therapeutic response appears to be similar [7, 9, 20-22], possibly reflecting the central importance of genioglossus stimulation and the favourable functional effects of the bilateral stimulation offered by the Genio ${ }^{\mathrm{TM}}$ system, as discussed in the previous paragraph.

The third unique feature of the Genio ${ }^{\mathrm{TM}}$ system is that the small stimulation unit, incorporating electrodes and a receiver, is the only implanted component and has no battery. It is implanted via a short midline submental incision and positioned proximate to the distal hypoglossal nerves. This electrode is activated transdermally by an activation unit that is worn externally. This differs from other devices which require surgical implantation of a unilateral cuff electrode around the hypoglossal nerve via a lateral submandibular incision which, in turn, are connected by at least one lead, which is tunnelled subcutaneously, to the implanted stimulator powered by an internal non-rechargeable battery and, in some of the HNS systems, to implanted respiratory sensing leads [7, 9, 12]. While no direct comparisons between systems have yet been made, it is possible that patients undergoing HNS using the Genio ${ }^{\mathrm{TM}}$ system will have relatively shorter surgery time, smaller and fewer incisions, faster healing time and less procedure-related post-operative pain. The external stimulation unit of the Genio ${ }^{\mathrm{TM}}$ system can be serviced readily, while servicing of the implanted stimulators of all these other systems (for example, for battery or system malfunction or device or firmware upgrade) would require explantation of the unit.

The fourth difference between the Genio ${ }^{\mathrm{TM}}$ system compared to others is that it delivers intermittent stimulation at a preprogrammed adjustable rate and duty cycle. Specifically, the system is programmed to deliver stimulation at a fixed rate, adjusted to be near the participant's own breathing frequency during unobstructed breathing when asleep. Stimulation duration is fully adjustable, but in the present trial was maintained at $70 \%$ of the total respiratory cycle time for most participants. This lengthy duty cycle was adopted in the knowledge that the participant's own rate would vary overnight, but still ensure stimulation for at least part of each inspiration. The cyclical pauses between stimulations were provided to allow for rest periods between muscle contractions. As such, stimulation is not systematically synchronised with the participant's breathing frequency, with stimulation occurring at variable periods in the respiratory cycle depending on the breathing frequency at any given time. This differs from other devices which deliver intermittent stimulation synchronised with inspiration as detected by respiration sensing leads [7, 9] or by delivering near-constant stimulation by cycling stimulation between multiple electrode combinations [12]. Advantages of the dispensing with inspiratory synchronisation by the Genio ${ }^{\mathrm{TM}}$ system via its predetermined adjustable rate and duty cycle approach is removal of the need for implanted respiratory effort sensing leads which add complexity, invasiveness and vulnerability to failure.

Despite the marked difference in modes of delivery of HNS, the magnitude of therapeutic response between these different devices appears to be similar [7, 9, 20-22]. This may be surprising, given the common belief that the pharyngeal airway is most vulnerable to collapse at end-expiration when the calibre of airway is thought to be at its smallest [36-39]. However, this pattern is not always observed and several studies have reported substantial inter- and intra-subject variability in the relationship of pharyngeal cross-sectional area to phase of respiration $[36,37,40]$. It is likely that the specific relationship between airway size, collapsibility and respiratory phase is a consequence of the relative contributions of a number of factors including transmural pressure gradients; pharyngeal muscle recruitment; lung volume; airway anatomy; head, neck and body posture; and sleep state [37, 39, 41, 42], which will vary between and within individuals.

The study has several limitations. By design the study was observational and did not have a control group. However, given the promising results to date, the next step would be to undertake a larger trial with possible control group and longer-term follow-up to confirm the findings of the current study. While the study did not reach its predefined target AHI reduction of 15 events $\mathrm{h}^{-1}$ used to compute the study sample size, it should be noted this was used only for estimate statistical power and the study achieved a statistically significant reduction in AHI.

In conclusion, the BLAST OSA study has demonstrated the safety and performance of the Genio ${ }^{\mathrm{TM}}$ system, associated with high adherence in participants with moderate-to-severe OSA and who have either not tolerated, failed or refused PAP therapy. The study showed significant reduction of OSA severity and improvement of sleepiness and quality of life, while keeping an acceptable safety profile. These results are consistent with previously published HNS systems, from which the Genio ${ }^{\text {TM }}$ system offers distinct, 
potentially advantageous differences. These include 1) bilateral rather than unilateral HNS; 2) minimal implanted components which are battery-less, with the activation unit worn externally; and 3) stimulation provided at a predetermined, adjustable rate and duty cycle rather than requiring inspiratory synchronisation with attendant implanted sensing leads. All these changes act to decrease the complexity and invasiveness of HNS application and simplify and facilitate maintenance of the system. Our findings suggest that these simplifications have been made without compromising safety or effectiveness.

Given the results of this study, the limitations of the existing treatment options, and the negative health and wellbeing consequences of leaving significant OSA untreated, the Genio ${ }^{\mathrm{TM}}$ system may be considered as a valid treatment option to treat OSA in a targeted population.

Acknowledgements: The BLAST OSA investigator team wishes to acknowledge the support from the Nyxoah (Mont-Saint-Guibert, Belgium) team, including Lucie Mortiaux, Jean-Marie Neyens, Tommy Rabon, Charles Czank, William Johnston, Rémi Renard and Nathalie Gilat.

Conflict of interest: P.R. Eastwood reports support for undertaking trials from Nyxoah SA, during the conduct of the study; support for undertaking trials from Oventus Pty Ltd and Zelda Therapeutics, outside the submitted work. M. Barnes has nothing to disclose. S.G. MacKay reports grants from NHMRC and Garnett-Passe RWF (conjoint grant), outside the submitted work. J.R. Wheatley reports grants from Nyxoah, during the conduct of the study. D.R. Hillman reports support for undertaking trials from Nyxoah SA, during the conduct of the study; support for undertaking trials from Oventus Pty Ltd and Zelda Therapeutics, outside the submitted work. X-L. Nguyên reports grants from Serenity Medical Services, during the conduct of the study. R. Lewis reports personal fees from Nyxoah SA, during the conduct of the study. M.C. Campbell reports personal fees from Nyxoah, outside the submitted work. B. Pételle reports consultancy fees from Bluesom, support for meeting attendance from Nyxoah, outside the submitted work. J.H. Walsh reports support for undertaking trials from Nyxoah SA, during the conduct of the study; support for undertaking trials from Oventus Pty Ltd and Zelda Therapeutics, outside the submitted work. A.C. Jones reports personal fees from Nyxoah, during the conduct of the study. C.E. Palme reports personal fees from Nyoxah, during the conduct of the study. A. Bizon has nothing to disclose. N. Meslier has nothing to disclose. C. Bertolus has nothing to disclose. K.J. Maddison has undertaken sponsored research projects for Nyxoah Pty Ltd, during the conduct of the study; has undertaken sponsored research projects for Nyxoah Pty Ltd, Oventus Pty Ltd and Zelda Pty Ltd, and reports grants from Sir Charles Gairdner Hospital and Australian and New Zealand College of Anaesthetists, outside the submitted work. L. Laccourreye reports non-financial support from Medtronic France and Integra LifeSciences Services, outside the submitted work. G. Raux has nothing to disclose. K. Denoncin has nothing to disclose. V. Attali reports personal fees for consultancy from Nyxoah, during the conduct of the study; personal fees for lectures from Resmed, institutional fees for research from Imthera, outside the submitted work. F. Gagnadoux has nothing to disclose. S.H. Launois is an employee of Serenity Medical Services/Bioserenity, reports personal fees for lectures from Cidelec, travel and/or meeting expenses from Vitalaire, S2A Santé Asten La Poste and UCB Pharma, grants from Resmed, outside the submitted work.

Support statement: This study trial was funded by Nyxoah S.A. P.R. Eastwood is funded by a National Health and Medical Research Council Senior Research Fellowship (number 1136548). S.G. MacKay is a Chief Investigator on National Health and Medical Research Council of Australia Grant, 1059510; Australian New Zealand Clinical Trials Registry ACTRN12614000338662 and a Chief Investigator on a Garnett Passe Rodney Williams Foundation Conjoint Grant.

\section{References}

1 Franklin KA, Lindberg E. Obstructive sleep apnea is a common disorder in the population - a review on the epidemiology of sleep apnea. J Thorac Dis 2015; 7: 1311-1322.

2 Harris M, Glozier N, Ratnavadivel R, et al. Obstructive sleep apnea and depression. Sleep Med Rev 2009; 13: 437-444.

3 Sawyer AM, Gooneratne NS, Marcus CL, et al. A systematic review of CPAP adherence across age groups: clinical and empiric insights for developing CPAP adherence interventions. Sleep Med Rev 2011; 15: 343-356.

4 Sunwoo BY, Light M, Malhotra A. Strategies to augment adherence in the management of sleep-disordered breathing. Respirology 2019; in press [https://doi.org./10.1111/resp.13589].

5 Schwartz AR, Smith PL, Oliven A. Electrical stimulation of the hypoglossal nerve: a potential therapy. J Appl Physiol 2014; 116: 337-344.

6 Schwartz AR, Bennett ML, Smith PL, et al. Therapeutic electrical stimulation of the hypoglossal nerve in obstructive sleep apnea. Arch Otolaryngol Head Neck Surg 2001; 127: 1216-1223.

7 Eastwood PR, Barnes M, Walsh JH, et al. Treating obstructive sleep apnea with hypoglossal nerve stimulation. Sleep 2011; 34: 1479-1486.

8 Malhotra A. Hypoglossal-nerve stimulation for obstructive sleep apnea. N Engl J Med 2014; 370: 170-171.

9 Strollo PJ Jr, Soose RJ, Maurer JT, et al. Upper-airway stimulation for obstructive sleep apnea. N Engl J Med 2014; 370: 139-149.

10 Woodson BT, Strohl KP, Soose RJ, et al. Upper airway stimulation for obstructive sleep apnea: 5-year outcomes. Otolaryngol Head Neck Surg 2018; 159: 194-202.

11 Zaidi FN, Meadows P, Jacobowitz $\mathrm{O}$, et al. Tongue anatomy and physiology, the scientific basis for a novel targeted neurostimulation system designed for the treatment of obstructive sleep apnea. Neuromodulation 2013; 16: $376-386$.

12 Friedman M, Jacobowitz O, Hwang MS, et al. Targeted hypoglossal nerve stimulation for the treatment of obstructive sleep apnea: six-month results. Laryngoscope 2016; 126: 2618-2623.

13 Vanderveken OM, Maurer JT, Hohenhorst W, et al. Evaluation of drug-induced sleep endoscopy as a patient selection tool for implanted upper airway stimulation for obstructive sleep apnea. J Clin Sleep Med 2013; 9: 433-438. 
14 Sher AE, Schechtman KB, Piccirillo JF. The efficacy of surgical modifications of the upper airway in adults with obstructive sleep apnea syndrome. Sleep 1996; 19: 156-177.

15 BaHammam AS, Obeidat A, Barataman K, et al. A comparison between the AASM 2012 and 2007 definitions for detecting hypopnea. Sleep Breath 2014; 18: 767-773.

16 Kushida CA, Nichols DA, Holmes TH, et al. Effects of continuous positive airway pressure on neurocognitive function in obstructive sleep apnea patients: the Apnea Positive Pressure Long-term Efficacy Study (APPLES). Sleep 2012; 35: 1593-1602.

17 Rosen CL, Auckley D, Benca R, et al. A multisite randomized trial of portable sleep studies and positive airway pressure autotitration versus laboratory-based polysomnography for the diagnosis and treatment of obstructive sleep apnea: the HomePAP study. Sleep 2012; 35: 757-767.

18 Weaver TE, Grunstein RR. Adherence to continuous positive airway pressure therapy: the challenge to effective treatment. Proc Am Thorac Soc 2008; 5: 173-178.

19 Cistulli PA, Armitstead J, Pepin JL, et al. Short-term CPAP adherence in obstructive sleep apnea: a big data analysis using real world data. Sleep Med 2019; 59: 114-116.

20 Steffen A, Sommer JU, Hofauer B, et al. Outcome after one year of upper airway stimulation for obstructive sleep apnea in a multicenter German post-market study. Laryngoscope 2018; 128: 509-515.

21 Kezirian EJ, Goding GS Jr, Malhotra A, et al. Hypoglossal nerve stimulation improves obstructive sleep apnea: 12-month outcomes. J Sleep Res 2014; 23: 77-83.

22 Mwenge GB, Rombaux P, Dury M, et al. Targeted hypoglossal neurostimulation for obstructive sleep apnoea: a 1-year pilot study. Eur Respir J 2013; 41: 360-367.

23 U.S. Food \& Drug Administration. Inspire II Upper Airway Stimulator. Pre Market Approval Number P130008. Docket number 14M-0690. www.accessdata.fda.gov/scripts/cdrh/cfdocs/cfpma/pma.cfm?id=P130008.

24 Johns MW. Sensitivity and specificity of the multiple sleep latency test (MSLT), the maintenance of wakefulness test and the Epworth sleepiness scale: failure of the MSLT as a gold standard. J Sleep Res 2000; 9: 5-11.

25 Weaver TE, Maislin G, Dinges DF, et al. Relationship between hours of CPAP use and achieving normal levels of sleepiness and daily functioning. Sleep 2007; 30: 711-719.

26 Zhu Z, Hofauer B, Wirth M, et al. Selective upper airway stimulation in older patients. Respir Med 2018; 140: $77-81$.

27 Hofauer B, Philip P, Wirth M, et al. Effects of upper-airway stimulation on sleep architecture in patients with obstructive sleep apnea. Sleep Breath 2017; 21: 901-908.

28 Steffen A, Kilic A, König IR, et al. Tongue motion variability with changes of upper airway stimulation electrode configuration and effects on treatment outcomes. Laryngoscope 2018; 128: 1970-1976.

29 Heiser C, Maurer JT, Steffen A. Functional outcome of tongue motions with selective hypoglossal nerve stimulation in patients with obstructive sleep apnea. Sleep Breath 2016; 20: 553-560.

30 Heiser C, Thaler E, Boon M, et al. Updates of operative techniques for upper airway stimulation. Laryngoscope 2016; 126: Suppl. 7, S12-S16.

31 Kubin L, Jordan AS, Nicholas CL, et al. Crossed motor innervation of the base of human tongue. J Neurophysiol 2015; 113: 3499-3510.

32 Safiruddin F, Vanderveken OM, de Vries N, et al. Effect of upper-airway stimulation for obstructive sleep apnoea on airway dimensions. Eur Respir J 2015; 45: 129-138.

33 Heiser C, Edenharter G, Bas M, et al. Palatoglossus coupling in selective upper airway stimulation. Laryngoscope 2017; 127: E378-E383.

34 Hofauer B, Strohl K, Knopf A, et al. Sonographic evaluation of tongue motions during upper airway stimulation for obstructive sleep apnea - a pilot study. Sleep Breath 2017; 21: 101-107.

35 Heiser C. Advanced titration to treat a floppy epiglottis in selective upper airway stimulation. Laryngoscope 2016; 126: Suppl. 7, S22-S24.

36 Walsh JH, Leigh MS, Paduch A, et al. Evaluation of pharyngeal shape and size using anatomical optical coherence tomography in individuals with and without obstructive sleep apnoea. J Sleep Res 2008; 17: 230-238.

37 Morrell MJ, Badr MS. Effects of NREM sleep on dynamic within-breath changes in upper airway patency in humans. J Appl Physiol 1998; 84: 190-199.

38 Schwab RJ, Gefter WB, Pack AI, et al. Dynamic imaging of the upper airway during respiration in normal subjects. J Appl Physiol 1993; 74: 1504-1514.

39 Morrell MJ, Arabi Y, Zahn B, et al. Progressive retropalatal narrowing preceding obstructive apnea. Am J Respir Crit Care Med 1998; 158: 1974-1981.

40 Launois SH, Remsburg S, Yang WJ, et al. Relationship between velopharyngeal dimensions and palatal EMG during progressive hypercapnia. J Appl Physiol 1996; 80: 478-485.

41 Schneider H, Boudewyns A, Smith PL, et al. Modulation of upper airway collapsibility during sleep: influence of respiratory phase and flow regimen. J Appl Physiol 2002; 93: 1365-1376.

42 Heinzer RC, Stanchina ML, Malhotra A, et al. Effect of increased lung volume on sleep disordered breathing in patients with sleep apnoea. Thorax 2006; 61: 435-439. 